Classification

Physics Abstracts

$42.30 \mathrm{Kq}-42.30 \mathrm{Sy}-62.20 \mathrm{Fe}$

\title{
Optical Diffraction of Stretched Lacunary Networks with Central Forces and Bond Bending Couplings
}

\author{
Pascal Monceau $\left({ }^{1,3}\right)$, Brice Barret $\left({ }^{2}\right)$, Denise Berger $\left({ }^{2}\right)$ and Jean-Claude Serge \\ Levy $\left({ }^{1}\right)$
}

(1) Laboratoire de Magnétisme des Surfaces, Université Parıs 7, 75251 Paris Cedex 05, France

$\left({ }^{2}\right)$ Laboratoire d'Optique Physıque, ESPCI, 10 rue Vauquelin, 75005 Paris, France

$\left({ }^{3}\right)$ Université d'Evry Val d'Essonne, Boulevard des Coquibus, 91025 Evry, France

(Received 27 October 1994, revised 13 March 1995, accepted 12 April 1995)

\begin{abstract}
Résumé. - Aprés avoir simulé numérıquement l'étirement d'un réseau aléatoire de sites reliés par des forces centrales entre sites occupés et par des forces proportionnelles aux écarts angulaires entre liaisons réalisées, la concentration en site étant proche de celle au seull de percolation, on étudie analytiquement et expérimentalement la diffraction optique d'un tel réseau déformé. Aux bords du réseau, les atomes sont solidaires du déplacement imposé, tandis qu'à l'intérieur différentes rotations apparaissent et sont détectées par diffraction. Ces régions internes, indépendantes, sont limitées par des pivots dont l'observation est usuelle dans les phénomènes de percolation. Les mesures dans l'espace direct, comme dans l'espace réciproque, de l'amplitude de ces rotations correspondent aux déductions théoriques qu'on peut mener à partir du modèle. L'étirement global résulte de la coordination de multiples rotations locales et donc la rigıdité effective du milieu est très faible.
\end{abstract}

\begin{abstract}
A numerically simulated network of randomly occupied sites connected by central forces and bond-bending couplings, whose the site concentration is slightly higher than the percolation threshold, is submitted to an external stretch. The optical diffraction pattern of the relaxed network is experumentally and analytically studied. In real space, regions at the boundary are shifted without being deformed, while most of the internal regions are rotated by some angles. Internal regions, bounded by pivots as usual in percolation theory are homogeneously rotated. These rotations are optically detected in reciprocal space and compared with the predictions of the theoretical model. Most of the stretching occurs by means of nearly free, independent rotations, without energy expense and thus with a very low bulk modulus.
\end{abstract}

\section{Introduction}

Elasticity in lacunary materials, or randomly distributed binary alloys, near the percolation threshold, has a large interest because of numerous practical applications such as the elasticity of rocks and cements [1], textile fibers [2] or polymers [3], for instance. Yet, due to the lack of 
translational and rotational invariance, a real theoretical difficulty is involved in the analytical treatment of the elastic properties of such materials. As a result, numerical studies are required, with the difficulty of choosing a both simple and generic model. For instance, when restricting considerations to a bidimensional case as is done here, the simple model of central forces on a square lattice which was considered previously by us $[4,5]$, is far from being generic since the percolation threshold in the infinite limit occurs at a concentration equal to one, i.e., a quite dissymmetrical case. In the more generic case, with both central forces and bondbending forces, percolation occurs at the same geometric threshold as electrical or geometrical percolation. Recent computations of their elastic properties were achieved on small samples, with a quadratic Hamiltonian, by means of a numerical inversion of the dynamical matrix [6]. Such a computation, for samples of about $p_{c} N^{2}$ sites taken within a square of side $N$, requires the inversion of a $2 p_{\mathrm{c}} N^{2} \times 2 p_{\mathrm{c}} N^{2}$ matrix which becomes an inaccurate operation if $N$ is not quite small. Thus, in order to derive the equilibrium configuration under stretching for a large enough sample, a relaxation method which mimics the physical process is more convenient. Relaxation processes according to a "steepest descent" resolution have been used for numerous structures $[4,7,8]$. Here, because of the vicinity of the percolation threshold, the so-called critical slowing down of the relaxation process occurs as known both experimentally [9] and theoretically $[4,10]$. Practically, the convergence of the steepest descent resolution can be strongly improved by the use of a conjugate gradient technique in the case of a quadratic Hamiltonian which enables us to obtain the equilibrium configuration within a reasonable computational time [11], and this process is used here. Such calculations also enable one to derive observable statistical properties such as the average Young modulus of a lattice at a given concentration [11]. But it is also of interest to compare the direct results of this modelling with experimental observations. From this point of view, optical diffraction and more generally diffraction experiments with $\mathrm{X}$-rays, electrons or neutrons, have the advantage of being a study of the media as a whole which gives an interesting global non destructive response in the case of elastic stimuli such as stretch or tension, and thus are quite interesting. This defines the goal of this study: to obtain a result about the modelled elasticity which can be easily compared to experimental ones.

In the case of interest here, of a random lacunary square network with both central forces between nearest neighbors and bond bending interactions, displacements, i.e., translations and rotations, preserve both nearest neighbor distance and bond angles, and are thus energetically free to occur. For this reason, the stretching of such materals will occur mainly from the combination of such displacements for some regions, 1.e., most of the sample, and unavordable distortion of transition regions where the elastic energy is concentrated. Of course, at these weaker places, also called pivots in the classical percolation theory [12], finite deformations of distance between nearest neighbors and of angles between nearest bonds occur. Thus, in such a bidimensional stretched lacunary network, four kinds of elastic regions appear :

- region of the first kind (I) is practically solidly fixed to one moving boundary, the left one or the right one, without any change relatively to this boundary.

- region $i$ of the second kind (II) is displaced during the relaxation process with a translational motion of vector $\mathbf{b}_{\imath}$ and a rotation of angle $\alpha_{\imath}$ around a center $O_{\imath}$.

- region of the third kind (III) is a small region connected to two regions (I) or (II) with different rotations, acting as a gearing. Such a restricted transition region experiments inhomogeneous distortion and is centred on a pivot, i.e., a site which, if unoccupied, would give rise to a lower connected state of at least one of its two neighboring regions [12]. 




Fig. 1. - The 620 sites, i e. diffracting pupils of the configuration $R(32,404)$.

- region of the fourth kind (IV) is independent from boundaries and thus remains still, without any motion, 1 e., does not even follow the motions of boundaries.

In this paper, the angles of rotation of regions (II) are approximately deduced, from a geometrical consideration on the location of their pivots and on the amplitude of stretching, and are also observed in the diffraction pattern of the whole sample. These results derived for optical diffraction can be easily extended to X-ray or electronic diffraction in a kinematic approach. Generalization to 3D problems is discussed.

The first section is devoted to an introduction of the elasticity problem in lacunary networks, while the second section deals with diffraction patterns observed from these targets under stretching.

\section{Elasticity in Lacunary Networks}

Here, a random network on a square lattice is considered: a configuration $R(n, q)$ where $p=$ $n^{2}-q\left(p \leq n^{2}\right)$ occupied sites are randomly chosen among the $n^{2}$ ones of a full square of side length $n$. In the configuration $R(32,404)$ of Figure 1 , which is studied here, the $p=620$ occupied sites define a site concentration $p / n^{2}=0.6054$, which is slightly higher than the geometrical percolation threshold of a square lattice $p_{c} 0.5927$ [12]. After getting rid of inert regions IV, there are still 561 active occupied sites in this configuration, as shown in Figure 2. Between each pair of neighbourng sites $i$ and $j$, i.e., distant of $a$ - the lattice parameter - at rest, a spring of stiffness $k$ is introduced with the local spring Hamiltonian $H_{2 j}^{\mathbf{s}}$ :

$$
H_{\imath \jmath}^{\mathrm{s}}=\frac{1}{2}\left(\left\|\mathbf{r}_{\imath}-\mathbf{r}_{\jmath}\right\|-a\right)^{2}
$$

where $\mathbf{r}_{i}$ defines the position of site $i$. Introducing the displacement vector $\mathbf{u}_{2}=\mathbf{r}_{2}-\mathbf{R}_{2}$ with respect to the rest position $\mathbf{R}_{\imath}$, and the parallel displacement $\left(\mathbf{u}_{i}-\mathbf{u}_{3}\right)_{\|}$which is the projection of the displacement vector $\left(\mathbf{u}_{2}-\mathbf{u}_{\jmath}\right.$ ) onto the vector $\mathbf{R}_{i}-\mathbf{R}_{\text {j }}$ at rest and of length $a$, the first non-constant term in the series development of the total spring Hamltonian $H^{\mathbf{s}}$ are quadratic in parallel displacements only and define a quadratic spring Hamiltonian $H^{\mathbf{s}, 2}[4,5]$ : 




Fig. 2. - The remainng 561 sites of Figure 1 after eliminating inert regions.

$$
H^{\mathrm{s}, 2}=\frac{1}{2} k \sum\left(\mathbf{u}_{\imath}-\mathbf{u}_{\jmath}\right)_{\|}^{2}
$$

with the spring constant $k$.

The local bond bending interaction $H^{\mathrm{bb}}$ is defined from the angle $\theta_{\imath \jmath k}$ between two sides $j i$ and $j k$ which share a common occupied site $j$, while neighbouring sites $i$ and $k$ are also both occupied [13]:

$$
H^{\mathrm{bb}}=(1 / 8) K \sin ^{2} 2 \theta
$$

with the angular spring constant $K$. Restricting this Hamiltonian to the part of the interaction which is quadratic in atomic displacements, we can write

$H_{\imath \jmath k}^{\mathrm{bb}, 2}=\frac{1}{2} K\left(x_{\imath}+x_{k}-2 x_{\jmath}\right)^{2}$, if both sides $i j$ and $j k$ are parallel to the $y$-axis

and

$H_{2 \jmath k}^{\mathrm{bb}, 2}=\frac{1}{2} K\left(x_{\imath}+y_{k}-x_{\jmath}-y_{\imath}\right)^{2}$, if side $i j$ is parallel to the $y$-axis, while side $j k$ is parallel to the $x$-axis.

Similar equations are easily derıved for other local arrangements by a standard application of symmetry rules. It can be noticed that this interaction links neighbors which can be next nearest ones if the angle $i j k$ is right and third ones if the angle $i j k$ is stralght. Thus, this interaction is already extended. In other words which refer to bonds, equations (4) mean that only perpendicular displacements are active for this interaction. As a consequence, the harmonic elastic coupling including central forces and bond bending interactions implies the solidarity of clusters of occupied sites connected by nearest neighbors since both parallel and perpendicular displacements of active bonds act.

The imposed stretch is defined by a given displacement of sites which are located on two opposite edges of the basic square for $R(n, q)$. The state of equlibrium of the network is 


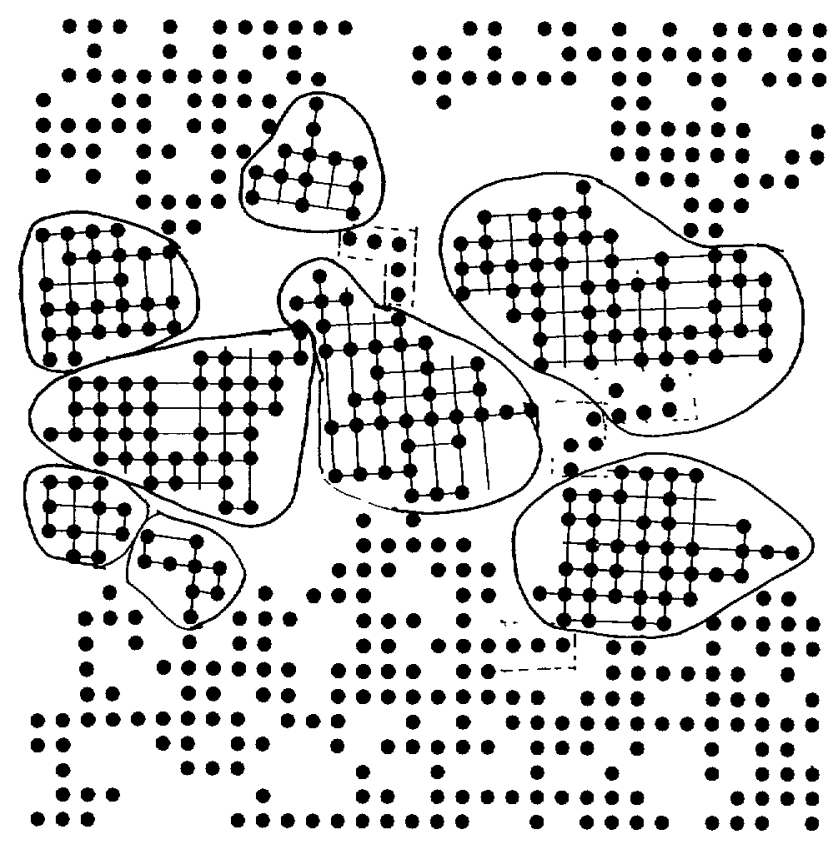

Fig. 3. - The equilibrium, after relaxation, of the 561 sites of Figure 2 when submitted to a shift of $0.3 a$ on lateral sides. Regions (II) are surrounded with continuous lines and are internally outlined by a frame of perpendicular lines, while regions (III) are surrounded with dotted lines.

reached at the end of a relaxation process $[4,5]$ which is accelerated here by the use of a conjugate gradient technique [11]. The standard relaxation process consists in calculating the forces $\mathbf{F}_{i}$ on each site and displacing each bulk atom by a displacement $\mathbf{u}_{i}=l \mathbf{F}_{\imath}$ where $l$ is a convenient damping parameter [4,7]. After each run over all mobile occupied sites the total elastic energy $E$ of the system is calculated; such runs are repeated until the variation of elastic energy between two successive runs is perfectly negligible. With the conjugate gradient technique, the atomic displacement $\mathbf{u}_{\imath}=l \mathbf{F}_{\imath}+m \mathbf{u}_{\imath-1}$ results from a linear combination of a displacement along the force and a displacement proportional to the previous displacement. The optimization of this more sophisticated process is done at each step and it ensures a rapid convergence. The numerical results are the same for both methods, but the conjugate gradient technique is quite faster than the standard method.

In Figure 3, the result of a stretch of amplitude $\delta=0.6 a$ applied to the configuration shown in Figures 1 and 2, when $(k=2 ; K=0.1)$, is plotted. Of course, the calculation has been restricted to active sites only, i.e., to the 561 ones of Figure 2. Moreover, in order to obtain a large displacement of a few percent of the initial scale, for the sake of an easy and accurate observation by diffraction techniques, several successive small displacements were done, taking as initial configuration the ending one of the previous displacement. The intermediate results were also treated and proved the linearity of these elastic perturbations. Similarly different ratios of bond bending versus string coupling were tried $(k=0.5 ; K=0.1)$ and $(k=0.5 ; K=0.8)$. The values of the interaction parameters which were taken for the sample shown in Figure 3 give rise to the largest motion. Thus the plotted results of Figure 3 give some large displacements which easily visualize the partition into regions (I) to (III). Different regions (II) and (III) appear. Regions (II) are surrounded by continuous lines and exhibit 


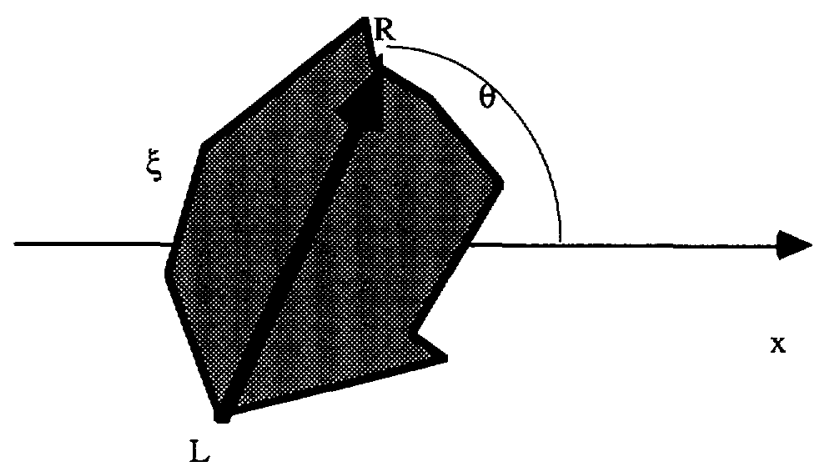

Fig. 4. - The scheme of a region (II) with its two pivots $L$ and $R$, and the orientation of the vector LR compared with that of the displacement axis $x$.

different angles of rotation, while regions (III) are surrounded by straight broken lines. In the internal part of each of the eight regions (II), narrow straight lines were added in order to connect sites on the same array, when at rest, and to visualize the bulk rotation of these regions under stretch. As displacements are rather large, a site overlapping happens between two sites belonging to different regions (II), without any real meaning in the computation since the overlapping sites were not neighbors at rest, and thus did not interact together as the interaction is left unchanged during the relaxation process. This accidental meeting of two sites emphasizes the observation of different rotation directions, as well as of different rotation intensities.

Usually from one boundary to the other one, there are independent parallel chains of regions (II), i.e., blocks and these blocks are connected by transition regions (III). This is the classical picture of nodes-links-blobs [14]. Of course this simple scheme is only valid as an approximation since a block can be connected with more than two other ones, in this case it means that these extra connections are weaker than the main two and can be neglected in a first step. Within this scheme, rotational angles can be estimated easily in a small or large sample.

First let us consider a small sample as schematized in Figure 4 when the stretching action occurs between the left hand side and the right hand side along the $x$-axis. Region II is bounded on the left by a pivot $L$ which connects this region to its left surrounding, and on the right by a pivot $R$ which connects this region to its right surrounding. The vector $\mathbf{L R}$ has for length $\xi$, the average size of a block, and makes an angle $\theta$ with the $x$-axis. There are only statistical constraints on this angle $\theta$ and it belongs to $[0,2 \pi]$. Thus the length of the projection of onto the $x$-axis is $\xi \cos \theta$. When this region is rotated by an angle $\mathrm{d} \theta$, the length of the projection of LR onto the $x$-axis is changed by $\delta=-\xi \sin \theta \mathrm{d} \theta$. Quite obviously, this variation $\delta$ corresponds to the displacement of the boundaries. Thus, for a given displacement $\delta$, the rotation angle is equal to $\mathrm{d} \theta=-\delta /(\xi \sin \theta)$ which is proportional to the stretching amplitude and inversely proportional to the block size and strongly depends on the orientation of the vector LR. This feature explains the possibility of the two directions of rotation and the numerous distinct rotations. The minimal amplitude of rotation is obtained for $\theta=\pi / 2$, with the value when $\delta=0.6 a ; \xi \approx 9 a: \mathrm{d} \theta \approx 4^{\circ}$ which is typical of the observations of Figure 3 where the effective dilation of a region is lower than that used in this calculation, but where the value of $\sin \theta$ is significantly lower than unity in such a way that these corrections balance each other.

Now considering a large sample of size $l$, a typical chain connecting both ends contains 


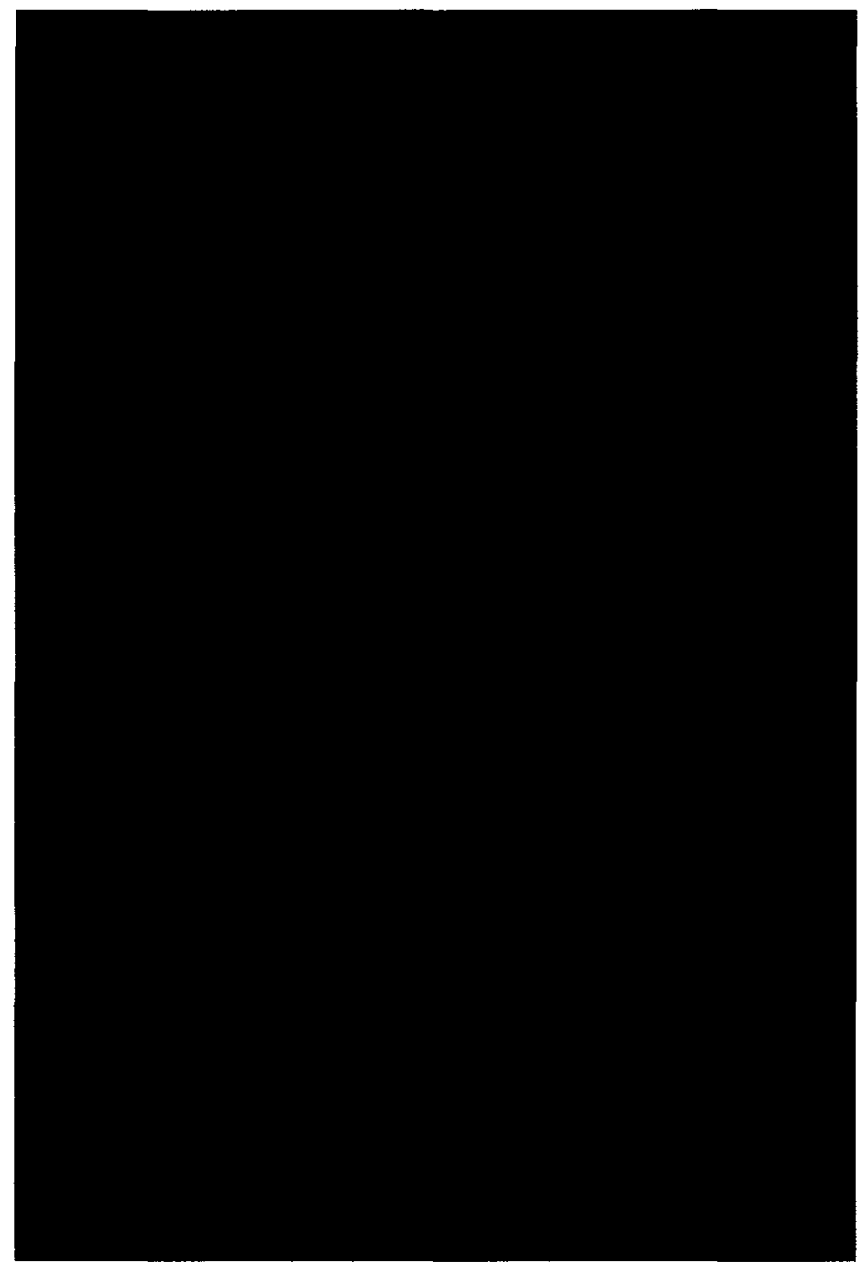

Fig. 5. - Optical diffraction patterns obtained from the pupıls shown in Figure 3. Several peaks can be distingushed at each order and especially at large orders $n$ where the shifts $n \delta \theta / a$ become large enough for separation of peaks.

$n=l^{2} / \xi^{2}$ regions (II) because of the law of large numbers, with an average orientation angle $\theta$ with the $x$-axis which is not very different from $\theta=\pi / 6$. These regions are connected by numerous transition regions (III). Thus the average change $\delta \theta$ of orientation of a region (II) is, when considering all changes as equal, a typical mean field approximation,

$$
\delta \theta \approx \frac{\delta / n}{\xi \sin \theta} \approx 2 \frac{2 \delta \xi}{l^{2}}
$$

Comparing this result to the observation, this change of orientation is obviously underestimated since numerous vectors $\mathbf{L R}$ have an inclination $\theta$ of a lower value and all displacements are not equal. Of course regions for which $|\sin \theta|$ is near unity are easily rotated with an effective large shift at the expense of a weak rotation angle. For an improved version, when taking as an average value $\overline{\sin \theta} \approx 0.2$, a correct value is found for the mean change of orientation. 
Table I. - Measured rotation angles in real and reciprocal space for different stretched samples, and nature of the diffraction spots.

\begin{tabular}{|c|c|c|c|}
\hline System & direct angle $\left(^{\circ}\right)$ & recip angle $\left(^{\circ}\right)$ & spots \\
\hline rel 13 & +0.5 & $+0.8 \_1.1$ & doublets \\
& -0.4 & $-0.5 \_0.9$ & \\
\hline relp 12 & +0.6 & $+0.6 \_0.9$ & doublets \\
& -0.5 & $-0.5 \_0.8$ & \\
\hline rel 3 & +0.9 & $+0.9 \_1.2$ & doublets \\
& -0.7 & $-0.7 \_1.1$ & triplets \\
\hline rel 12 & +1.2 & $+1.3 \_1.6$ & triplets \\
& -0.8 & $-1.1 \_1.4$ & \\
\hline str 13 & +1.9 & $+1.8 \_2.4$ & quadruplets \\
& -1.5 & $-1.2 \_1.8$ & \\
\hline str 12 & +2.7 & $+2.1 \_2.6$ & quadruplets \\
& -1.9 & $-1.9 \_2.4$ & \\
\hline
\end{tabular}

\section{Diffraction on Stretched Lacunary Networks}

The optical diffraction by photographs of free or stretched lacunary spring networks is realized in the classical Fraunhofer method $[5,15]$ with for pupil, a copy of a bidimensional network which is illuminated under normal incidence by an extended laser beam. The diffracted pattern is observed in the focal plane FT (Oxy) of a lens of focal distance $f$ as shown in Figure 4 of reference [5]. Practically, the image in plane FT is projected onto screen $\mathrm{S}$ by means of a lens which enables an enlargement of this image. The diffraction pattern of networks $R(n, q)$ at rest, with a majority of occupied sites, is quite similar to those of complete networks since, basically, the same main local structures arise in both networks. Of course, it results from a convolution of factors relative to the beam extension, to the pattern of sites and to the shape of a site. Here, the circular shape of a site implies the occurrence of Airy functions in the diffraction patterns, and this modulation can be changed by changing the size of individual sites.

The similarity between the diffraction patterns of lacunary random networks and of full networks was also observed in the diffraction patterns of rather dense deterministic fractals $[16,17]$. Thus this diffraction pattern is expected to consist of a few points which are due to the square lattice matrix and of a rather extended central isotropic spot which is due to random lacunae, in a state of disorder over long distances. More precisely, the diffraction pattern depends upon the nature of a site: full or not, square or circle and of its size [5,16]. Here, in the copy submitted to diffraction, an occupied site is represented by a full small circle with a diameter $c$ which is one tenth of the distance $a$ between neighbouring sites. This ensures that an Airy diffraction pattern is obtained for sites with a central spot which is several times larger than the parameter $b$ of the square lattice in the reciprocal space [15], while the full extension 


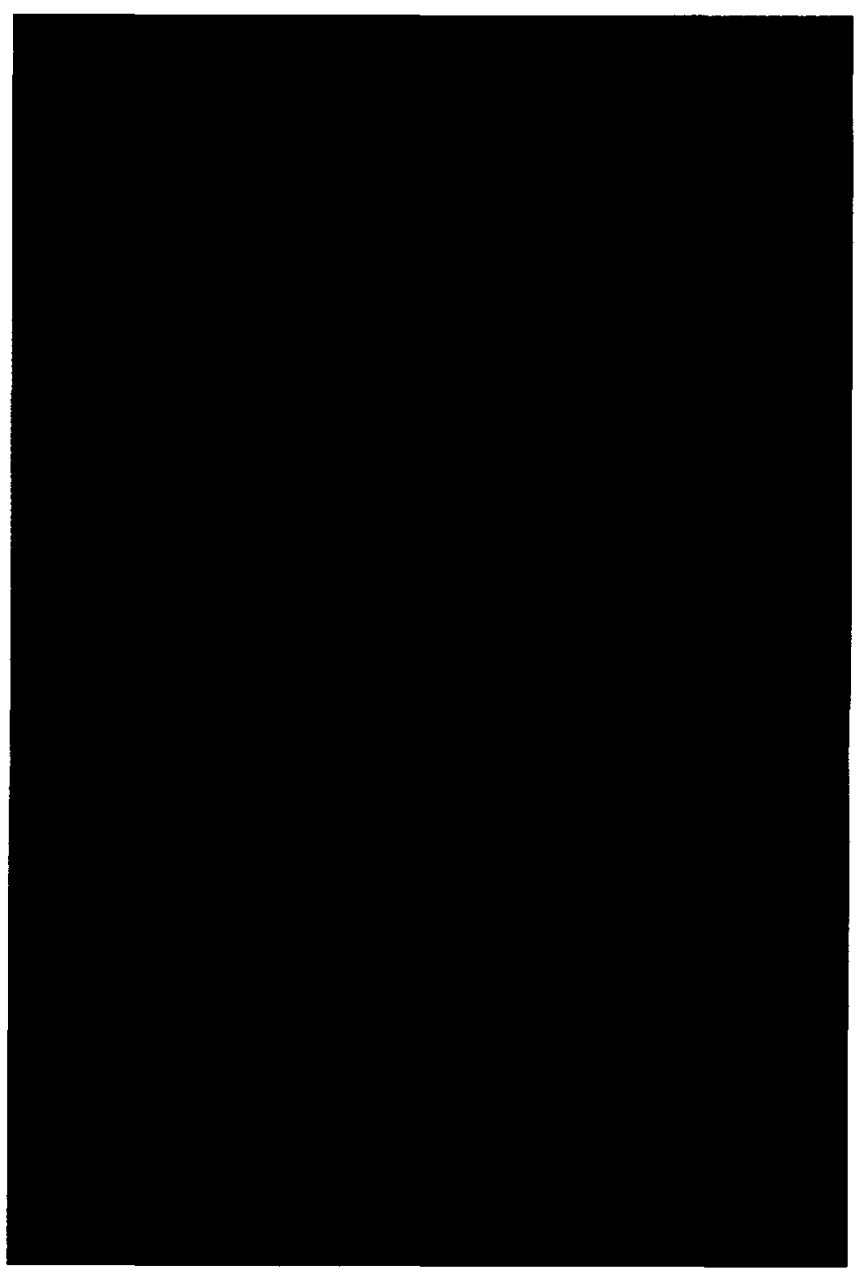

Fig. 6. - Diffraction patterns obtained from the pupls of Figure 3 which are included within a rectangular mask. One main peak appears at each level while other peaks associated with unperfectly masked other regions II have a smaller intensity.

of the laser beam is larger than that of the copy of the network, which provides a correct resolution. The diffraction patterns of similar lacunary networks at rest, or stretched, but with springs only, have been investigated previously [5] and the analysis of the diffraction pattern under stretching enabled the authors to distinguish several regions and their translational motion by means of a vernier effect.

Considering the diffraction by a network of points $i$ with a coherent incldent radiation of wavevector $\mathbf{k}(0,0, k)$ and a diffracted radiation of wavevector $\mathbf{k}^{\prime}(k x / f, k y / f, k)$, where Gauss conditions imply $x, y \ll f$, with $f$ for focal length of the lens, the diffraction wavevector $\mathbf{S}$ is: $\mathbf{S}=\mathbf{k}^{\prime}-\mathbf{k}$ and the diffracted intensity $I$ reads :

$$
I=\sum_{i, 3} \exp \left(i \mathbf{S} \cdot \mathbf{r}_{\imath \jmath}\right)=\sum_{i \jmath} \exp \left(i \mathbf{S}\left[\mathbf{r}_{3}-\mathbf{r}_{2}\right]\right.
$$


the sum is extended over the sites $i$, with the vector $\mathbf{r}_{\imath}=\mathbf{O M}_{\imath}$ defining the site $i$ in the target plane Oxy.

In the pupil shown in Figure 3 there are two regions (I), i.e., undeformed but just shifted, each one at a shifted boundary. Each of these regions (I) gives rise to approximately the diffraction pattern of a perfect square lattice, as already noticed. The interference effect between these two regions must give rise to an extra modulation due to the vernier effect well studied previously [5]. However, since sites belonging to these regions (I) are not so numerous, this effect of modulation is not so important as in the previous configuration. Most of the sites belong to regions (II) which thus give the main contribution to the diffraction pattern. The diffraction pattern of a single region (II) is similar to that of a region (I) which has a similar topology. More exactly since any region (II) can be deduced from a region (I) by a convenient rotation $R_{\varphi}$ in real space, its diffraction pattern is deduced from that of this region (I) by a rotation $R_{\varphi}$ in reciprocal space, so as to maintain the scalar product $\left(i \mathbf{S} \cdot\left[\mathbf{r}_{j}-\mathbf{r}_{\imath}\right]\right)$ constant for each pair of lattice sites of the considered region, of course these rotation centers lie in different spaces, but their angles $\varphi$ are equal. In a more detailed manner, when introducing the rotation matrix $\mathbf{R}_{\varphi}$ in real space, the rotated pair of lattice sites $\mathbf{r}_{\mathfrak{\jmath}}^{\prime}-\mathbf{r}_{k}^{\prime}$ is now written

$$
\mathbf{r}_{j}^{\prime}-\mathbf{r}_{i}^{\prime}=\mathbf{R}_{\varphi} \quad\left(\mathbf{r}_{j}-\mathbf{r}_{\imath}\right)
$$

The same rotation for the line dual vector $\mathbf{S}$ reads, because of the unitary nature of rotation matrices:

$$
\mathbf{S}^{\prime}=\mathbf{S} \cdot{ }^{t} \mathbf{R}_{\varphi}=\mathbf{S} \cdot \mathbf{R}_{\varphi}^{-1}
$$

Finally the scalar product $\left(i \mathbf{S} \cdot\left[\mathbf{r}_{j}-\mathbf{r}_{i}\right]\right)$ remains invariant when the same rotations are made simultaneously in real and reciprocal space with:

$$
\mathbf{S}^{\prime} \cdot\left[\mathbf{r}_{\jmath}^{\prime}-\mathbf{r}_{i}^{\prime}\right]=\mathbf{S} \cdot \mathbf{R}_{\varphi}^{-1} \cdot \mathbf{R}_{\varphi} \cdot\left[\mathbf{r}_{\jmath}-\mathbf{r}_{\imath}\right]=\mathbf{S} \cdot\left[\mathbf{r}_{\jmath}-\mathbf{r}_{i}\right]
$$

Thus, several different rotations of the pattern observed for regions (I) are expected to occur in the diffraction pattern of the whole sample, eight according to the analysis of Figure 3, with an average amplitude of a few degrees as considered previously. It can be observed on the numerous diffraction spots at several order that several rotations of a few degrees actually occur in the diffraction pattern shown in Figure 5. These rotations are obvious at all orders of diffraction, practically on more than one hundred spots and can be easily measured, even if the accuracy of these measurements of small angles is not perfect. Several systems with different elastic parameters and with different stretches were studied both in real space and in reciprocal space, and the results of the comparison between angles of rotation in real and reciprocal space are reported in Table I. Extreme rotations in direct and reverse geometrical direction are noted in degrees. There is a strong simllarity between the measured angles in both spaces. Since these angles are rather small and not perfectly defined even in real space because the boundary between region (II) and region (III) cannot be exactly given, these results give an idea of the validity of this partition into distinct regions and of the definition of these angles within a few percent.

About interference effects between different regions (II), it must be outlined that rotational motions are rather complexly imbricated and thus do not induce specific effects but an increase of diffusion as noticed experimentally. The relation between one region and a specific rotation in the Fourier space can be emphasized by using masks on the copy submitted to the laser beam. This is done in the case of Figure 6, where a mask selects one region and its surrounding. Thus the diffraction pattern with this mask looks like that of an individual region but with the presence of small tracks of other rotations which are still present because of the imperfect 
masking and of a rather large diffusion due to the relative increase of the contribution of transition regions (III).

\section{Conclusions}

From this work on optical diffraction from pupils deduced from numerical simulation of stretch in lacunary materials with central forces, as well as bond bending ones, it appears that most of the displacement is associated with internal rotations of numerous independent parts called blobs. The rotation angles can be measured accurately by means of this technique, i.e., with an accuracy of a few percent which is their optimal definition. Such an evidence of rotations was already predicted in the case of polymers, with the introduction of the word "strophons" to call such local rotations [3]. Here the interest lies in the non destructive method of diffraction for observing these elastic properties.

Several generalizations of this idea of elasticity by means of complementary rotations are quite tempting. First of all, the change of scale to deal with atomic motions is quite obvious to deduce, within the kinematical theory of diffraction and leads to a change of wavelength, i.e., to deal with $\mathrm{X}$-rays and electronic diffraction. Another interesting generalization consists in dealıng with actual motions, i.e., in following the motions induced by the action of a variable imposed displacement. Precisely here such dynamic effects were not involved, but considering a quasistatic approach defined by nearly continuous states of equlibrium, rotational motions are easily deduced, their inertial properties must be of interest in this combination of rotating motions.

Quite obviously, the generalization of such static or dynamic rotational displacements to a three-dimensional space seems also to be quite interesting. But these three-dimensional displacements require a careful statistical analysis, because many rotations around axes with different orientations are involved at the same time.

\section{Acknowledgments}

The authors are glad to acknowledge Dr M. Perreau for his invaluable help.

\section{References}

[1] Webman I., Fractals in Physics, L. Pietronero and E. Tosatti, Eds. (Elsevier, Amsterdam, 1986) p. 343.

[2] Oudet C., Polymères, structure et propriétés (Masson, Parıs, 1994).

[3] Yannas I.V. and Luise R.R., The Strength and Stiffness of Polymers, A.E. Zachariades and R.S. Porter Eds. (Marcel Dekker, New York, 1983) p. 255.

[4] Monceau P.J.-M. and Lévy J.C.S., Phys. Rev. B 49 (1994) 1026.

[5] Monceau P.J.-M., Lévy J.C.S., Isambert H. and Berger D., J. Phys. I France 3 (1993) 1891.

[6] Feng S., Sen P.N., Halperın B.I. and Lobb C.J., Phys. Rev. B 30 (1984) 5386; Feng S. and Sahimı M., Phys. Rev. B 31 (1985) 1671; Feng S., Phys. Rev. B 32 (1985) 510; ibid. 5793.

[7] Mercier D. and Lévy J.C.S., Phys. Rev. B 27 (1983) 1292.

[8] Roth J., Schilling R. and Trebin H.R., Phys. Rev. B 41 (1990) 2735.

[9] Ngar K.L., Mundy J.N., Jain H., Balzer-Jöllenbeck G. and Kanert O., Phys. Rev. B 39 (1989) 6071 . 
[10] Nakayama T., Yakubo K. and Orbach R.L., Rev. Mod. Phys. 66 (1994) 381.

[11] Monceau P.J.-M., Thesıs, Université Parıs 7 (1994); Monceau P.J.-M. and Lévy J.C.S., submitted to publication.

[12] Grimmett G., Percolation (Sprınger-Verlag, New York, 1989).

[13] Webmann I., Fractals in Physics, L. Pietronero and E. Tossati, Eds. (Elsevier, Amsterdam, 1986).

[14] Skal A.S. and Shklovskii B.I., Sov. Phys. Semicond. 8 (1975) 1029; de Gennes P.G., J. Phys. Paris 37 (1976) L1.

[15] Hecht E., Optics (Addison Wesley, 2nd edition, Reading, Mass., 1987).

[16] Kaye B.H., A Random Walk Through Fractal Dimensıon (V.C.H. Verlag, Berlin, 1989).

[17] Allain C. and Cloitre M., Physica A 157 (1989) 352. 